\title{
Simultaneous targeting of EGFR and mTOR inhibits the growth of colorectal carcinoma cells
}

\author{
BO LI $^{1}$, SHUOHUI GAO ${ }^{1}$, FENG WEI $^{2}$, ANITA C. BELLAIL ${ }^{2}$, CHUNHAI HAO $^{2}$ and TONGJUN LIU ${ }^{1}$ \\ ${ }^{1}$ Department of Colorectal Surgery, The Third Hospital of Jilin University, Changchun, Jilin 130033, P.R. China; \\ ${ }^{2}$ Department of Pathology and Laboratory Medicine, Emory University School Medicine, Atlanta, GA 30329, USA
}

Received January 17, 2012; Accepted March 5, 2012

DOI: $10.3892 /$ or.2012.1786

\begin{abstract}
Epidermal growth factor receptor (EGFR) is highly expressed in colorectal carcinomas and, as a result, it leads to the activation of downstream mammalian target of rapamycin (mTOR) kinase pathways for cancer growth and progression. Clinical and preclinical studies, however, have shown that inhibition of epidermal growth factor receptor (EGFR) and mammalian target of rapamycin (mTOR) alone is not sufficient to treat colorectal carcinomas. In search of effective combination therapies, we show here that simultaneous targeting of EGFR with its inhibitor, erlotinib and mTOR with its inhibitor, rapamycin inhibits the phosphorylation and activation of downstream phosphatidylinositol 3-kinase (PI3K), Akt, mTOR and extracellular-signal-regulated kinase 1/2 (Erk1/2) pathways, resulting in the inhibition of cell cycle progression and the growth of both KRAS wild-type and mutated colorectal carcinoma cells. This study has demonstrated the principle that the combination of erlotinib and rapamycin may provide an effective therapy for colorectal carcinomas.
\end{abstract}

\section{Introduction}

Colorectal carcinoma is one of the most common cancers and the fourth leading cause of cancer-related deaths worldwide (1). Studies of cancer signaling pathways have resulted in the generation of novel therapeutic agents that target the signaling pathways for the treatment of colorectal carcinomas (2). Epidermal growth factor receptor (EGFR), a member of

Correspondence to: Dr Tongjun Liu, Department of Colorectal Surgery, The Third Hospital of Jilin University, 126 Xiantai Street, Changchun, Jilin 130033, P.R. China

E-mail: tongjunliu2010@hotmail.com

Dr Chunhai Hao, Department of Pathology and Laboratory Medicine, Emory University School of Medicine, Atlanta, GA 30329, USA

E-mail: chao@emory.edu

Key words: epidermal growth factor receptor, mammalian target of rapamycin, targeted therapy, colorectal carcinoma, cell cycle the receptor tyrosine kinase family is highly expressed in colorectal carcinomas, resulting in the activation of downstream cell growth pathways involved in cancer growth and progression (3). EGFR can be therapeutically targeted by at least two approaches: i) small molecular inhibitors such as gefitinib and erlotinib that interfere with the ATP-binding site on EGFR and ii) neutralizing antibodies including cetuximab and panitumumab that target the extracellular domain of EGFR and interfere with ligand binding of the receptor (4). Clinical trials of the antibodies have shown some response in approximately $10 \%$ of patients with metastatic colorectal carcinomas (5-7) and lung carcinoma association with EGFR amplification, but not mutations (5). In contrast, gefitinib and erlotinib have shown no such response in clinical trials (8). The mechanisms of cancer resistance to EGFR-targeted therapeutics include the activation of redundant downstream kinase pathways in colorectal carcinoma. Current efforts have therefore focused on development of combination therapies targeting the redundant cell growth pathways in colorectal carcinomas $(9,10)$.

EGFR can activate downstream phosphatidylinositol 3-kinase (PI3K) and Akt through interaction with intracellular adaptors, growth factor receptor-bound protein 2 (Grb2) and Grb2-associate binder 1 (Gab1), driving cancer progression (11) and metastasis (12). In addition, point mutations in the p110 $\alpha$ subunit of PI3K have been identified in colorectal carcinomas (13) and the mutated (mt) p110 $\alpha$ can directly activate the PI3K/Akt pathway (14). EGFR can also activate the mitogenactivated protein kinase/extracellular-signal-regulated kinase 1/2 (Erk1/2) pathway through the small G-protein, RAS (15). $K R A S$ is a human homologue of the Kirsten rate sarcoma-2 virus oncogene and its mutation in colorectal carcinomas correlates to poor prognosis of the cancer patients (16). Colorectal carcinomas with wild-type (wt) KRAS respond to panitumumab better than KRAS-mutated (mt) carcinomas (17). The American Society of Clinical Oncology has suggested testing KRAS mutations in colorectal carcinomas to predict their response to EGFR antibodies (18).

Mammalian target of rapamycin (mTOR) is a serine and threonine kinase and downstream effector of the PI3K/Akt pathway that links EGFR with cell growth $(19,20)$. mTOR exists in two distinct functional complexes: mTORC1 and mTORC2 (21). mTORC1 controls protein translation through the phosphorylation and activation of eukaryotic translational 
initiation factor 4E (eIF4E) binding protein (4E-BP1) and p70S6 ribosomal kinase (S6K) (22). mTORC1 is sensitive to rapamycin treatment (21) whereas mTORC2 is insensitive to rapamycin except with prolonged treatment (23). The rapamycin analog, RAD-001 is currently in clinical trials for colorectal carcinoma therapy; however, a recent study suggests that targeting of mTOR alone may not be sufficient enough to inhibit cancer growth because inhibition of mTOR activates the PI3K/Akt pathway for cancer growth (24). In this study, we show that combination treatment with the EGFR inhibitor, erlotinib, and the mTOR inhibitor, rapamycin, inhibits the PI3K/Akt, Erk1/2 and mTOR pathways and thereby the cell cycle progression of colorectal carcinoma cells.

\section{Materials and methods}

Reagents and antibodies. Erlotinib and rapamycin were purchased from LC Laboratories (Woburn, MA) and prepared as stock solutions in DMSO. Epidermal growth factor (EGF) was purchased from Peprotech (Rocky Hill, NJ). The antibodies to EGFR, phospho-EGFR (Tyr1068), mTOR, phosphor-mTOR (Ser2448), Akt, phosphor-Akt (Ser473), and ribosomal protein S6 (rpS6), phosphor-rpS6 (Ser235/236), Erk1/2 and phosphorErk1/2 (Thr202/Tyr204) were from Cell Signaling Technology (Danvers, MA). Horseradish peroxidase (HRP)-conjugated goat anti-mouse and goat anti-rabbit antibodies were from Jackson IR Laboratories (West Grove, PA). Protease inhibitor mixture, Triton X-100 and other chemicals were purchased from Sigma-Aldrich. The chemiluminescence detection system was from Amersham Biosciences (Piscataway, NJ).

Colorectal carcinoma cell lines. Human colorectal carcinoma cell lines DLD-1, HCT-8, HT-29 and HCT-116 were purchased from American Type Culture Collection (ATCC; Rockville, MD). DLD-1 and HCT-8 were grown in RPMI-1640 medium (Invitrogen, Carlsbad, CA) and HT-29 and HCT-116 were cultured in McCoy's 5A medium (Invitrogen). The medium was supplemented with $10 \%$ fetal bovine serum (FBS) and $1 \%$ penicillin-streptomycin. Cells were maintained in a humidified $5 \% \mathrm{CO}_{2}$ and $37^{\circ} \mathrm{C}$ incubator.

Cell viability assay. Cell viability was determined by the acid phosphatase assay (25). In brief, cells were plated in a 96-well plate at $8 \times 10^{3}$ cells/well in $200 \mu \mathrm{l}$ of $10 \%$ FBS-containing medium. After 24-h incubation, the medium was replaced with $1 \%$ FBS medium with erlotinib or vehicle (DMSO) in the presence or absence of EGF $(50 \mathrm{ng} / \mathrm{ml})$. After incubation for the times indicated in the Results, cells were washed with phosphate-buffered saline (PBS) and $100 \mu \mathrm{l}$ buffer containing $0.2 \mathrm{M}$ sodium acetate $(\mathrm{pH} 5.5), 0.2 \%(\mathrm{v} / \mathrm{v})$ Triton $\mathrm{X}-100$ and $20 \mathrm{mM}$ p-nitrophenyl phosphate (Sigma 104 phosphatase substrate) was added to each well. The plates were incubated at $37^{\circ} \mathrm{C}$ for $1.5 \mathrm{~h}$ and the reaction was stopped by the addition of $10 \mu 11 \mathrm{M} \mathrm{NaOH}$ to each well and the color developed was measured at $405 \mathrm{~nm}$ by a microplate reader (Bio-Rad Laboratories, Hercules, CA).

Flow cytometry analysis of the cell cycle. Cells were seeded in 6 -well plates at a density of $5 \times 10^{5}$ cells/well. After $24 \mathrm{~h}$ incubation, cells were grown for $24 \mathrm{~h}$ in $1 \%$ FBS medium supplemented with or without EGF $(50 \mathrm{ng} / \mathrm{ml})$ in the presence or absence of DMSO as a control, erlotinib (1 $\mu \mathrm{M})$, and/or rapamycin $(100 \mathrm{nM})$. After treatment, cells were harvested, washed with PBS, and fixed by incubation in $1 \%$ paraformaldehyde and then in $70 \%$ ethanol solution at $4^{\circ} \mathrm{C}$. The fixed cells were washed and the washed cell pellets were stained using a propidium iodide-RNase solution (PBS containing $20 \mu \mathrm{g} / \mathrm{ml}$ propidium iodide, $20 \mu \mathrm{g} / \mathrm{ml}$ DNase-free RNase A, and $0.1 \%$ Triton X-100) for $30 \mathrm{~min}$ at $20^{\circ} \mathrm{C}$ in the dark. The cell cycle status was analyzed with a flow cytometer using the ModFit LT software (Verity Software House Inc., Topsham, ME).

Western blotting. Western blotting was carried out based on the protocols reported earlier (25). In brief, cells were harvested in lysis buffer consisting of $20 \mathrm{mM}$ Tris $\mathrm{pH} 7.4,150 \mathrm{mM} \mathrm{NaCl}$, $1 \%$ NP-40, 10\% glycerol, 1 mM EGTA, 1 mM EDTA, $5 \mathrm{Mm}$ sodium pyrophosphate, $50 \mathrm{mM}$ sodium fluoride, $10 \mathrm{mM}$ $\beta$-glycerophosphate, $1 \mathrm{mM}$ sodium vanadate, $0.5 \mathrm{mM}$ DTT, $1 \mathrm{mM}$ PMSF, $2 \mathrm{mM}$ imidazole, $1.15 \mathrm{mM}$ sodium molybdate, $4 \mathrm{mM}$ sodium tartrate dihydrate, and $1 \mathrm{X}$ protease inhibitor cocktail (Sigma). Following 30-min incubation in lysis buffer at $4^{\circ} \mathrm{C}$, lysates were cleared by centrifugation at $18,000 \mathrm{x} \mathrm{g}$ $15 \min 4^{\circ} \mathrm{C}$. The supernatant was collected and protein concentrations were determined by the Bradford protein assay following the manufacturer's protocol (Bio-Rad Laboratories). Equal amounts of protein were separated through SDS-PAGE gels and transferred onto nitrocellulose membranes (Bio-Rad Laboratories). The membranes were incubated overnight at $4^{\circ} \mathrm{C}$ with a primary antibody and then for $1 \mathrm{~h}$ with an HRP-conjugated secondary antibody. The membranes were developed by chemiluminescence.

Statistical analysis. Data are presented as means \pm standard deviation (SD) and were analyzed statistically by the t-test.

\section{Results}

Erlotinib inhibits the EGFR downstream PI3K/Akt, Erk and mTOR pathways. Upon EGF binding, EGFR is phosphorylated and activated, leading to the activation of the downstream,PI3K/ Akt, mTOR and Erk1/2 kinases pathways. To examine which of the kinase pathways are activated by EGFR in colorectal carcinomas, we grew colorectal carcinoma cell lines in $1 \%$ FBS culture medium containing $50 \mathrm{ng} / \mathrm{ml}$ of EGF. To determine whether KRAS mutations affect the kinase pathways, we selected colorectal carcinoma cell lines that have been found to carry either wt KRAS (HT-29, HCT-8) and mt KRAS (DLD-1, HCT-116) (26). After $24 \mathrm{~h}$ in culture, the cells were lysed and examined by western blotting for the phosphorylation of EGFR and downstream kinases. Non-phosphorylated EGFR, Akt, mTOR, and Erk1/2 were detected in the cells before and after EGF treatment in KRAS wt HT-29 and HCT-8 cells (Fig. 1A); the results suggest that EGF does not stimulate the expression of the kinase proteins. In contrast, however, phosphorylated (p-) EGFR, p-Akt, p-mTOR and p-Erk1/2 were detected in the cells treated with EGF (Fig. 1A), suggesting that EGF stimulates the phosphorylation and activation of EGFR and downstream kinases, PI3K/Akt, mTOR and Erk1/2 in KRAS wt colorectal carcinoma cells. To determine whether inhibition of EGFR can block EGF-stimulated downstream kinases, 
A

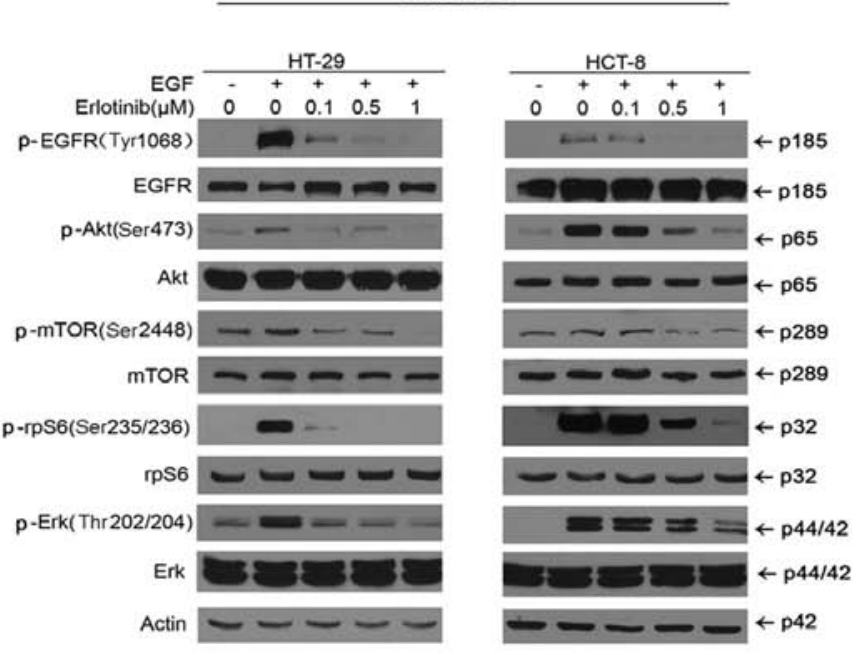

B

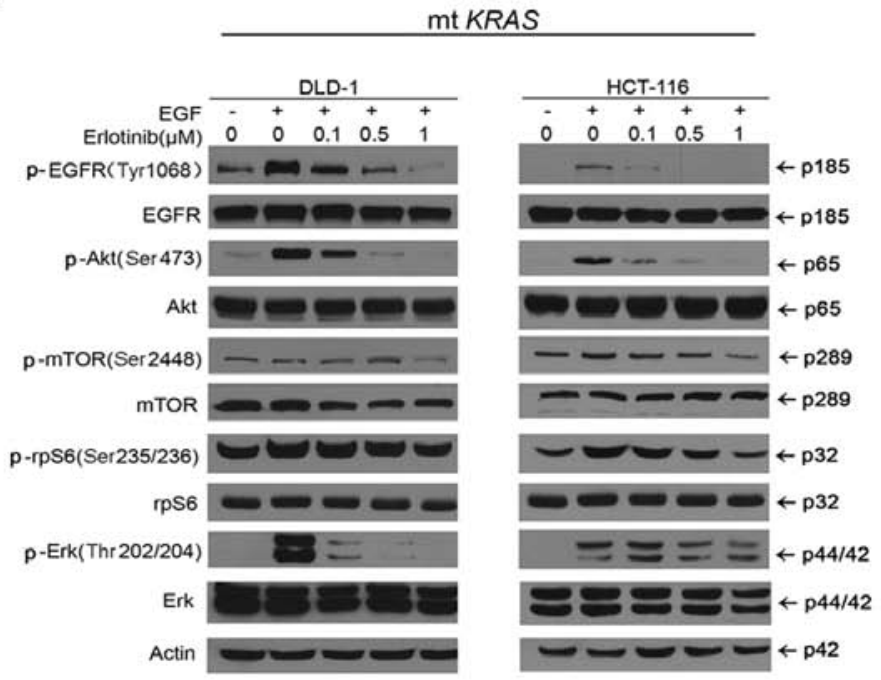

Figure 1. Erlotinib treatment inhibits EGFR downstream kinase pathways. Colorectal carcinoma cell lines with wild-type (wt) KRAS (A) and mutant (mt) $K R A S(\mathrm{~B})$, as indicated on the top of the panel, were untreated or treated with $50 \mathrm{ng} / \mathrm{ml}$ of EGF and erlotinib in the doses as indicated on the top of the panels. The cells were then examined by western blotting with antibodies to the phosphorylated (p-) and unphosphorylated EGFR and downstream kinase pathway proteins as indicated to the left of the panels with their molecular weights indicated to the right of the panels. Actin was used as a protein-loading control.

we treated the cells with various doses of the EGFR inhibitor, erlotinib. Western blot analysis showed that treatment with erlotinib significantly reduced p-EGFR, p-Akt, p-mTOR and p-rpS6 in these cell lines (Fig. 1A). These data suggest that inhibition of EGFR can block EGF-induced phosphorylation and activation of EGFR and downstream PI3K/Akt, mTOR and Erk1/2 in KRAS wt colorectal carcinoma cells.

Prior to EGF treatment KRAS mt DLD-1 and HCT-116, cells expressed p-mTOR and p-rpS6 but not p-Akt and p-Erk1/2 (Fig. 1B). This suggests that the mTOR pathway is constitutively activated in KRAS mutant colorectal carcinoma cells. This was also noticed in the KRAS wt HCT-8 cell line (Fig. 1A). These KRAS mt cell lines were then treated with EGF; western blots detected p-EGFR, p-Akt and p-Erk1/2 in the cell lines after EGF treatment, suggesting that EGF treatment activates the PI3K/Akt and Erk1/2 pathways in KRAS mt colorectal carcinoma cells. The experiment was repeated in the presence of erlotinib. Western blotting showed that erlotinib treatment led to the reduction of p-Akt and p-Erk1/2 but not p-mTOR and p-rpS6 in these KRAS mt cell lines (Fig. 1B). Taken together, the data suggest that inhibition of EGFR by erlotinib can block EGF-stimulated PI3K/Akt, Erk1/2 and mTOR in KRAS wt carcinoma cells but not of the mTOR pathway in KRAS mt carcinoma cells.

Erlotinib treatment inhibits cell cycle progression. The findings that erlotinib treatment blocks the activation of EGFR downstream kinase pathways suggest that erlotinib treatment may inhibit the growth of colorectal carcinoma cells. To test this hypothesis, we grew colorectal carcinoma cell lines in the presence of $50 \mathrm{ng} / \mathrm{ml}$ of EGF and various doses of erlotinib for $24 \mathrm{~h}$. Cell viability analysis showed that erlotinib treatment inhibited the growth of both KRAS wt and mt cell lines (Fig. 2A). To explore the molecular basis of erlotinib action, we treated these cell lines with erlotinib and analyzed the cell cycle of the treated cells. Flow cytometry analysis showed that erlotinib treatment resulted in a dramatic increase of the number of cells in the $G_{0} / G_{1}$ phase but a decrease of the number of cells in the $\mathrm{S}$ phase (Fig. 2B and C). The percentage of the cells in the $\mathrm{S}$ phases, was decreased from 22 to $12 \%$ in HT-29, from 18 to $4 \%$ in DLD-1, from 24 to $6 \%$ in HCT-8 and from 14 to $7 \%$ in HCT-116 cells. These results suggest that erlotinib treatment inhibits cell cycle progression of colorectal carcinoma cells regardless of KRAS status, consistent with the report from a clinical trial that $K R A S$ mutation has no effect on treatment of colorectal carcinomas by another EGFR inhibitor, gefitinib (8).

Inhibition of the mTOR pathway does not inhibit cell growth. The finding that mTOR is activated constitutively in three of the four colorectal carcinoma cell lines suggests the possibility that inhibition of mTOR may inhibit the growth of the cells. To test this hypothesis, we grew colorectal carcinoma cell lines for $24 \mathrm{~h}$ in medium containing $50 \mathrm{ng} / \mathrm{ml}$ EGF in the presence or absence of various doses of mTOR inhibitors, rapamycin. The cell viability assay revealed the rapamycin treatment did not significantly affect the growth of KRAS wt HT-29 and HCT-8 and KRAS mt DLD-1 and HCT-116 cell lines (Fig. 3A). Western blot analysis revealed a complete inhibition of p-rpS6 in KRAS wt HCT-8 cells and a partial inhibition of p-rpS6 in KRAS mt DLD-1 cells; however, p-Akt remained present and slightly increased in rapamycin-treated $K R A S$ wt and $\mathrm{mt}$ cell lines (Fig. 3B). This is consistent with a recent report that inhibition of mTOR may lead to activation of the PI3K/Akt pathway (24).

Simultaneous targeting of EGFR and mTOR inhibits cell growth. Inhibition of mTOR alone does not prevent the growth of colorectal carcinoma cells in part due to the activation of the PI3K and Erk1/2 pathway; thus, we thought to examine whether simultaneous targeting of EGFR and mTOR suppresses these kinase pathways and inhibits cancer growth. 
A

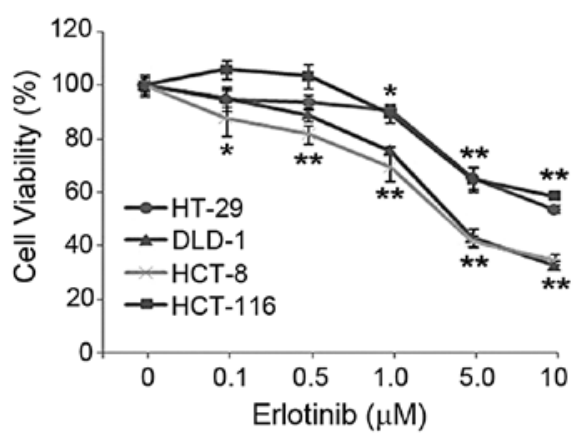

B
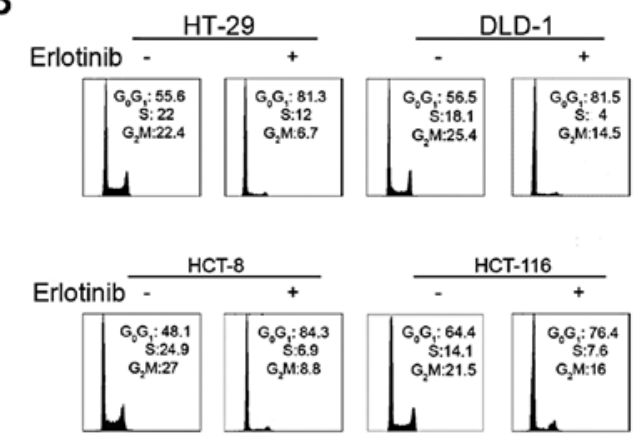

C

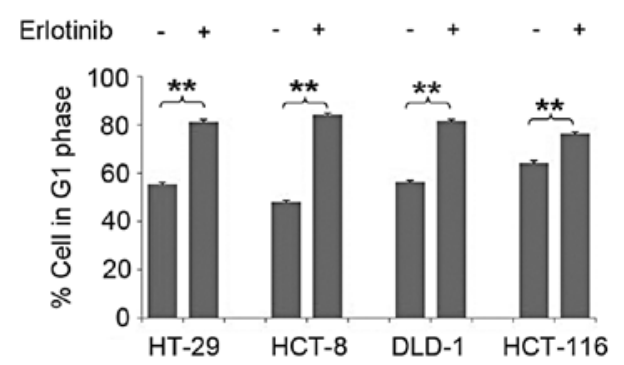

Figure 2. Erlotinib inhibits colorectal carcinoma cell growth. (A) Colorectal carcinoma cell lines were treated with various doses of erlotinib for $24 \mathrm{~h}$ and examined by cell viability of the inhibition of cell growth. The experiments were repeated three times and data were presented as mean $\pm \mathrm{SD}$ and analyzed statistically by the Student's t-test. ${ }^{*} \mathrm{P}<0.05 ;{ }^{* *} \mathrm{P}<0.001$. (B) These cell lines were treated with $1 \mu \mathrm{M}$ of erlotinib for $24 \mathrm{~h}$ and subjected to flow cytometry cell cycle analysis. The population of $\mathrm{G}_{0} / \mathrm{G}_{1}, \mathrm{~S}$ and $\mathrm{G}_{2} / \mathrm{M}$ phase cells are presented as percentages. (C) The experiments in (B) were repeated three times and the percentages of cells in the $G_{1}$ phase are presented as means \pm SD and analyzed statistically by the Student's t-test. ${ }^{* *} \mathrm{P}<0.001$.

To this end, we treated colorectal carcinoma cell lines with erlotinib and rapamycin, alone or in combination. The treated cells were further examined by a cell viability assay and the results showed that the combination treatment significantly inhibited the cell growth of all the cell lines as compared to erlotinib treatment alone (Fig. 4A). Flow cytometry analysis of the cell cycle further revealed that the combination treatment dramatically inhibited the cell cycle progression of both KRAS wt and mt cell lines (Fig. 4B and C). Western blot analysis showed that the combination treatment either eliminated or significantly reduced p-EGFR, p-Akt, p-mTOR, p-rpS6 and p-Erk1/2 expressed in both KRAS wt and mt cell lines (Fig. 5). These results suggest that simultaneous targeting of EGFR and mTOR can block the PI3K/Akt, Erk1/2 and mTOR pathways in colorectal carcinoma cells and thus inhibits the cell cycle progression of these cancer cells.
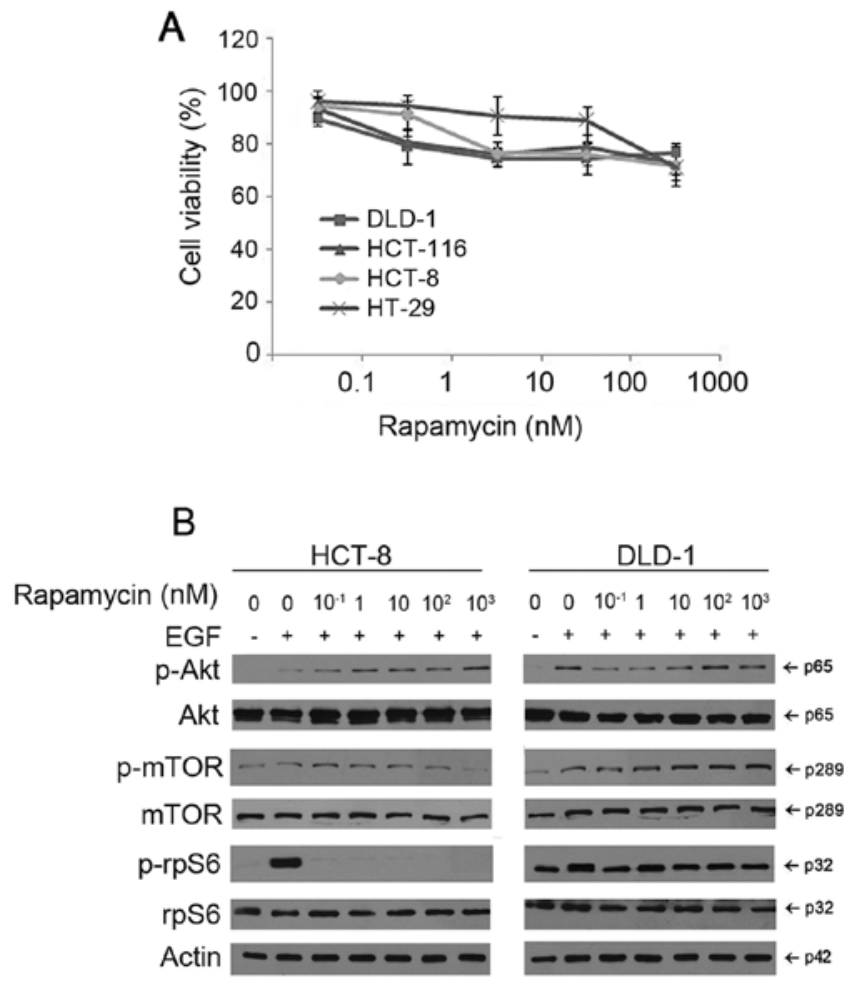

Figure 3. Rapamycin inhibits the mTOR pathway. (A) Colorectal carcinoma cell lines were treated with rapamycin with the doses indicated in the bottom of the panel and analyzed by a cell viability assay. The data are presented as means $\pm \mathrm{SD}$ and statistically analyzed. (B) KRAS wt HCT-8 and KRAS mt DLD-1 cell lines were treated with $50 \mathrm{ng} / \mathrm{ml}$ of EGF and various doses of rapamycin as indicated on the top of the panel for $24 \mathrm{~h}$. The untreated and treated cells were examined by western blotting with the antibodies to the proteins indicated on the left and molecular weights on the right of the panel.

\section{Discussion}

Recent advances in cancer biology have identified signaling pathways involved in the formation and progression of colorectal carcinomas, leading to the development of signaling pathway-targeted therapies for colorectal carcinomas (2). EGFR and mTOR are overexpressed in colorectal carcinomas $(3,27)$; however, targeted inhibition of EGFR and mTOR alone does not provide effective treatment of colorectal carcinomas $(8,24)$. The data presented here show for the first time that simultaneous inhibition of EGFR and mTOR results in inhibition of the phosphorylation and activation of PI3K/Akt, Erk1/2 and mTOR pathways in colorectal carcinoma cells, leading to the inhibition of the cell cycle progression and growth of colorectal carcinoma cells. The combination of EGFR and mTOR inhibitors may thus provide an effective treatment of carcinomas.

Stimulation of EGFR with EGF activates EGFR downstream cell growth pathways in colorectal carcinoma cells; however, inhibition of EGFR with its small molecular inhibitors and naturalizing antibodies as single agents has not shown any significant tumor response in patients with colorectal and lung cancers $(5-8,28)$. In this study, we show that EGFR stimulation can activate multiple cell growth pathways including PI3K/Akt, Erk1/2 and mTOR and thus drives cancer progression. Interestingly, mTOR is constitutively activated in one of 
A
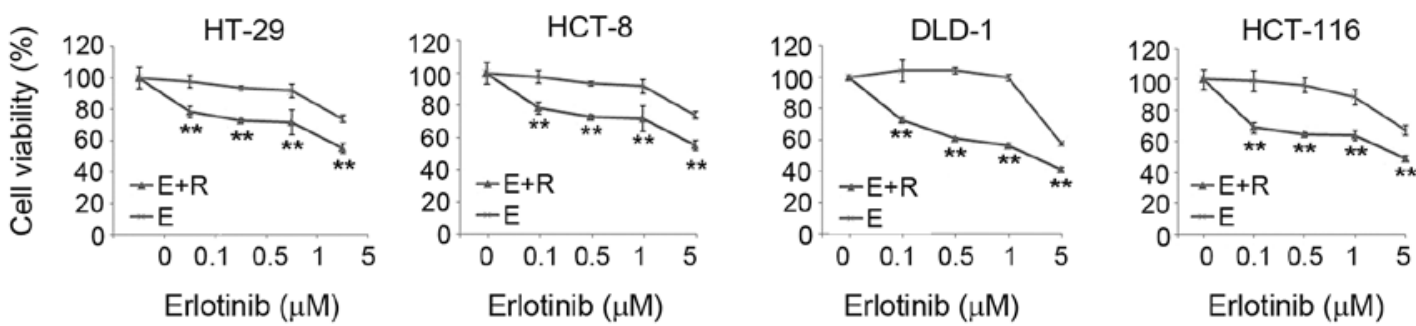

B

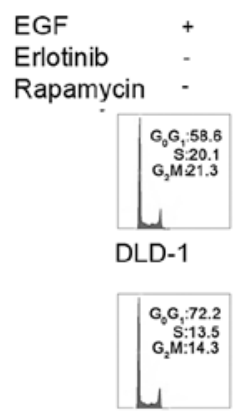

HCT-116
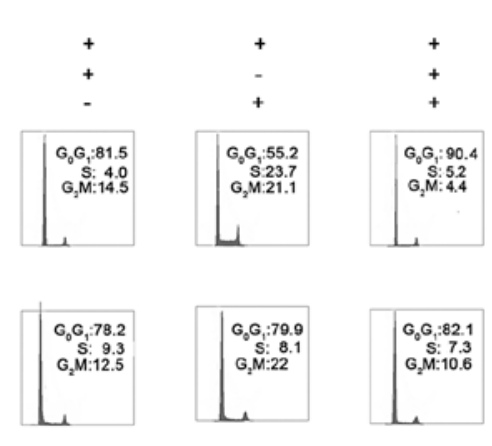

C

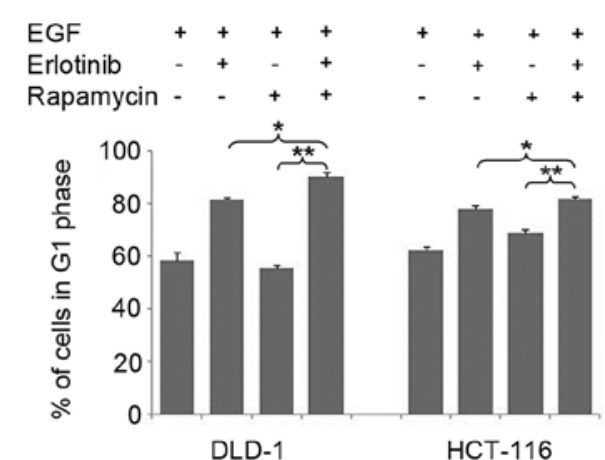

Figure 4. The combination of erlotinib and rapamycin inhibits cell growth. (A) Colorectal carcinoma cell lines were treated for $24 \mathrm{~h}$ with erlotinib (E) alone or in combination with $1 \mathrm{nM}$ of rapamycin $(\mathrm{E}+\mathrm{R})$. The cells were then analyzed by a cell viability assay. The data represent the mean $\pm \mathrm{SD}$ of three independent experiments. The Student's-test was used to analyze the data. ${ }^{* * *} \mathrm{P}<0.001$. (B) The KRAS mt DLD-1 and HCT-116 cell lines were treated with the combination of $1 \mathrm{nM}$ of rapamycin and $1 \mu \mathrm{M}$ of erlotinib for $24 \mathrm{~h}$ and the cell cycle distribution was examined by flow cytometry. The percentages of the cells in each of the cell cycle phases are indicated. (C) The experiments described in (B) were repeated three times and the percentage of cells in the $\mathrm{G}_{1}$ phase are presented as the mean \pm SD and analyzed by the Student's t-test. ${ }^{*} \mathrm{P}<0.05 ;{ }^{* *} \mathrm{P}<0.001$.
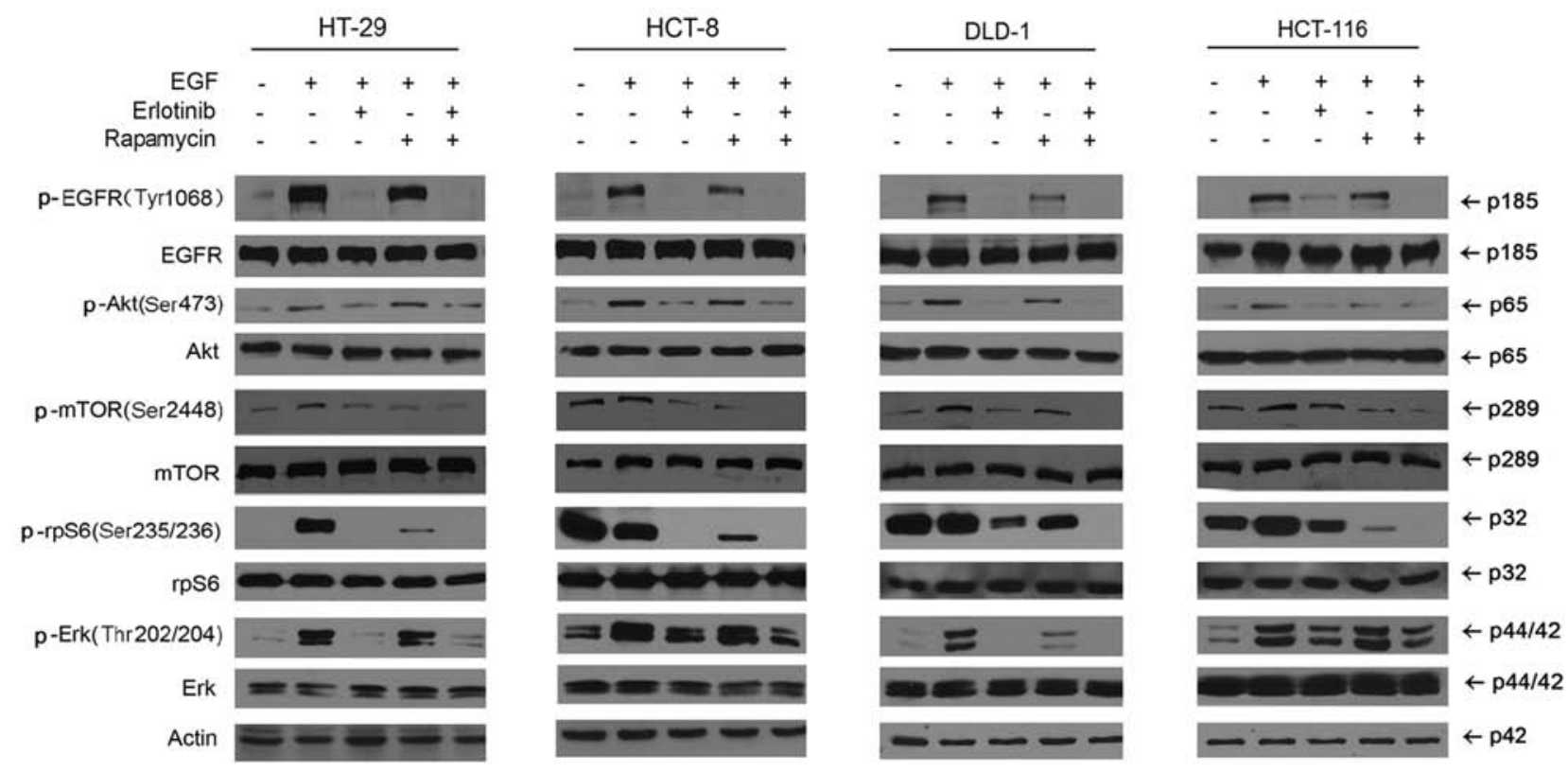

Figure 5. The combination of erlotinib and rapamycin inhibits multiple kinase pathways. Colorectal carcinoma cell lines were treated with $50 \mathrm{ng} / \mathrm{ml}$ of EGF in the presence or absence of $1 \mu \mathrm{M}$ of erlotinib or $1 \mathrm{nM}$ of rapamycin for $24 \mathrm{~h}$. The untreated and treated cells were examined by western blotting for the phosphorylated and unphosphorylated EGFR and kinases as indicated on the left of the panel.

the two KRAS wt but in two of two KRAS mutant colorectal carcinoma cells. Inhibition of EGFR can block the mTOR pathway in KRAS wt but not in KRAS mutant colorectal carcinoma cells.

There are two mTOR complexes and both of the complexes are overexpressed in colorectal carcinomas (27); however, treatment with the mTOR inhibitor, rapamycin fails to inhibit the growth of colorectal carcinoma cells as evident in this study. This is in part due to the activation of PI3K/Akt and Erk1/2 pathways that drive cancer progression, as shown in this study. In addition, rapamycin mainly targets the mTOR complex 1 (mTORC1) (21); however, a recent study shows that 
inhibition of mTORC2 results in the inhibition of tumorigenesis and progression of colorectal carcinoma (27). This study suggests that mTORC2 may be a therapeutic target in treating colorectal carcinomas; however, the distinguished functions of mTORC1 and mTORC2 in the content of PI3K/Akt pathways in colorectal carcinoma cells remain to be clarified.

Preclinical and clinical studies have proved that inhibition of EGFR, PI3K/Akt and Erk1/2 or mTOR alone is not sufficient to inhibit cancer cell growth. Combination therapies are needed to block the multiple kinase cell growth pathways for cancer treatment $(9,10)$. In this study, we show for the first time that the combination of the mTOR inhibitor, rapamycin, with the EGFR inhibitor, erlotinib, inhibits the phosphorylation and activation of multiple kinase pathways, the PI3K/Akt, Erk1/2 and the mTOR. This combination treatment leads to inhibition of cell cycle progression and growth of colorectal carcinoma cells. This study therefore suggests that simultaneous targeting of EGFR and mTOR may provide an effective treatment of colorectal carcinomas through inhibition of multiple kinase pathways.

\section{References}

1. Parkin DM, Bray F, Ferlay $\mathbf{J}$ and Pisani P: Global cancer statistics, 2002. CA Cancer J Clin 55: 74-108, 2005.

2. Meyerhardt JA and Mayer RJ: Systemic therapy for colorectal cancer. N Engl J Med 352: 476-487, 2005.

3. Huang SM and Harari PM: Epidermal growth factor receptor inhibition in cancer therapy: biology, rationale and preliminary clinical results. Invest New Drugs 17: 259-269, 1999.

4. Hynes NE and Lane HA: ERBB receptors and cancer: the complexity of targeted inhibitors. Nat Rev Cancer 5: 341-354, 2005.

5. Moroni M, Veronese S, Benvenuti S, et al: Gene copy number for epidermal growth factor receptor (EGFR) and clinical response to antiEGFR treatment in colorectal cancer: a cohort study. Lancet Oncol 6: 279-286, 2005.

6. Cunningham D, Humblet Y, Siena S, et al: Cetuximab monotherapy and cetuximab plus irinotecan in irinotecan-refractory metastatic colorectal cancer. N Engl J Med 351: 337-345, 2004.

7. Saltz LB, Meropol NJ, Loehrer PJ Sr, Needle MN, Kopit J and Mayer RJ: Phase II trial of cetuximab in patients with refractory colorectal cancer that expresses the epidermal growth factor receptor. J Clin Oncol 22: 1201-1208, 2004.

8. Rothenberg ML, LaFleur B, Levy DE, et al: Randomized phase II trial of the clinical and biological effects of two dose levels of gefitinib in patients with recurrent colorectal adenocarcinoma. J Clin Oncol 23: 9265-9274, 2005.

9. Hezel AF and Ryan DP: Emerging therapies for colorectal cancer. Expert Opin Investig Drugs 16: 867-876, 2007.
10. Zhang YJ, Tian XQ, Sun DF, Zhao SL, Xiong H and Fang JY: Combined inhibition of MEK and mTOR signaling inhibits initiation and progression of colorectal cancer. Cancer Invest 27: 273-285, 2009.

11. Lemmon MA and Schlessinger J: Cell signaling by receptor tyrosine kinases. Cell 141: 1117-1134, 2010.

12. Rychahou PG, Kang J, Gulhati P, et al: Akt 2 overexpression plays a critical role in the establishment of colorectal cancer metastasis. Proc Natl Acad Sci USA 105: 20315-20320, 2008.

13. Samuels Y, Wang Z, Bardelli A, et al: High frequency of mutations of the PIK3CA gene in human cancers. Science 304: $554,2004$.

14. Ikenoue T, Kanai F, Hikiba Y, et al: Functional analysis of PIK3CA gene mutations in human colorectal cancer. Cancer Res 65: 4562-4567, 2005.

15. Yarden Y and Sliwkowski MX: Untangling the ErbB signalling network. Nat Rev Mol Cell Biol 2: 127-137, 2001.

16. Esteller M, Gonzalez S, Risques RA, et al: K-ras and p16 aberrations confer poor prognosis in human colorectal cancer. J Clin Oncol 19: 299-304, 2001

17. Amado RG, Wolf M, Peeters M, et al: Wild-type KRAS is required for panitumumab efficacy in patients with metastatic colorectal cancer. J Clin Oncol 26: 1626-1634, 2008.

18. Allegra CJ, Jessup JM, Somerfield MR, et al: American Society of Clinical Oncology provisional clinical opinion: testing for KRAS gene mutations in patients with metastatic colorectal carcinoma to predict response to anti-epidermal growth factor receptor monoclonal antibody therapy. J Clin Oncol 27: 2091-2096, 2009.

19. Choo AY and Blenis J: TORgeting oncogene addiction for cancer therapy. Cancer Cell 9: 77-79, 2006.

20. Shaw RJ and Cantley LC: Ras, PI(3)K and mTOR signalling controls tumour cell growth. Nature 441: 424-430, 2006.

21. Guertin DA and Sabatini DM: Defining the role of mTOR in cancer. Cancer Cell 12: 9-22, 2007.

22. Ma XM and Blenis J: Molecular mechanisms of mTOR-mediated translational control. Nat Rev Mol Cell Biol 10: 307-318, 2009.

23. Sarbassov DD, Ali SM, Sengupta S, et al: Prolonged rapamycin treatment inhibits mTORC2 assembly and Akt/PKB. Mol Cell 22: $159-168,2006$

24. Hay N: The Akt-mTOR tango and its relevance to cancer. Cancer Cell 8: 179-183, 2005.

25. Lu G, Liu Y, Ji B, Wei F, Hao C and Wang G: Synergistic effect of celecoxib on TRAIL-induced apoptosis in hepatocellular carcinoma cells. Cancer Invest 28: 629-634, 2010.

26. Benvenuti S, Sartore-Bianchi A, Di Nicolantonio F, et al: Oncogenic activation of the RAS/RAF signaling pathway impairs the response of metastatic colorectal cancers to anti-epidermal growth factor receptor antibody therapies. Cancer Res 67: 2643-2648, 2007.

27. Gulhati P, Cai Q, Li J, et al: Targeted inhibition of mammalian target of rapamycin signaling inhibits tumorigenesis of colorectal cancer. Clin Cancer Res 15: 7207-7216, 2009.

28. Eberhard DA, Johnson BE, Amler LC, et al: Mutations in the epidermal growth factor receptor and in KRAS are predictive and prognostic indicators in patients with non-small-cell lung cancer treated with chemotherapy alone and in combination with erlotinib. J Clin Oncol 23: 5900-5909, 2005. 